\title{
Endoscopic choledochoenterostomy using a new blunt-ended endoscopic ultrasound-guided access device
}

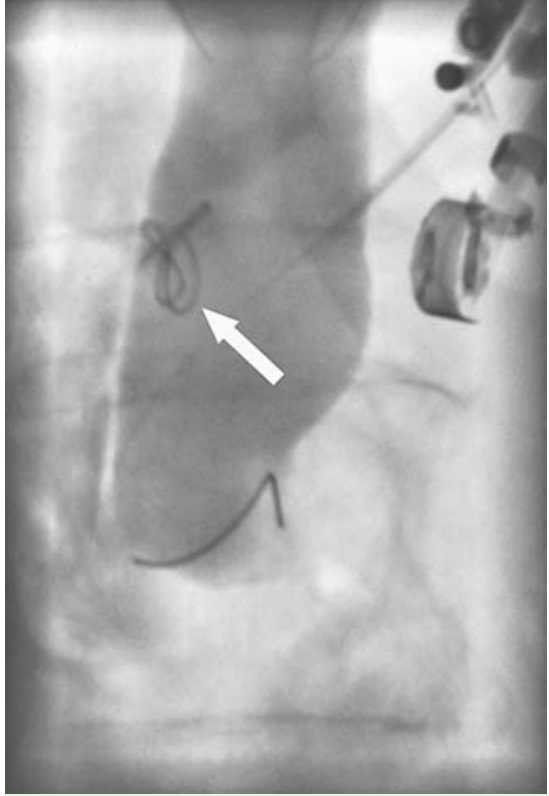

Fig. 1 Foreign body consisting of a wire-coating in the bile duct (arrow).

During wire manipulation in duct access procedures, conventional endoscopic ultrasound (EUS) needles can shear their wire coating ( $\bullet$ Fig. 1), as has been previously well documented in vascular procedures [1-3], thereby leaving a foreign body in the duct and/or making it difficult to retract the wire into the needle. The needle can also potentially cause trauma to the duct wall.

The following two brief cases represent early examples of using a novel bluntended access device ( $\bullet$ Fig. 2 ) that contains a sharp needle-tipped stylet (Cook Medical, Winston Salem, North Carolina, USA) to rescue failed biliary stenting.

The needle stylet is withdrawn after duct access has been obtained, and wire exchange is then done through the bluntended metal sheath, which has less potential for damaging the wire coating and the duct wall.

The first inpatient, an 84-year-old woman, had a circumferential duodenal adenocarcinoma involving the entire second part of the duodenum (T4 due to pancreatic invasion). The ampulla was lost in tumor and the duodenal lumen was too narrowed for a side-viewer; thus, a rendezvous procedure was infeasible. A forward-viewing

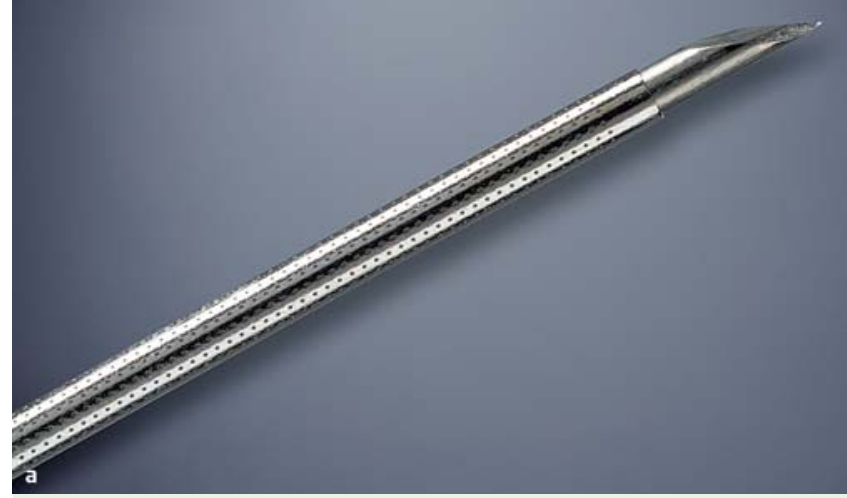

Fig. 2 Dimpled (echogenic) access metal sheath: a the needle-tipped sheath; and $\mathbf{b}$ with the sharp stylet removed (Cook Medical).
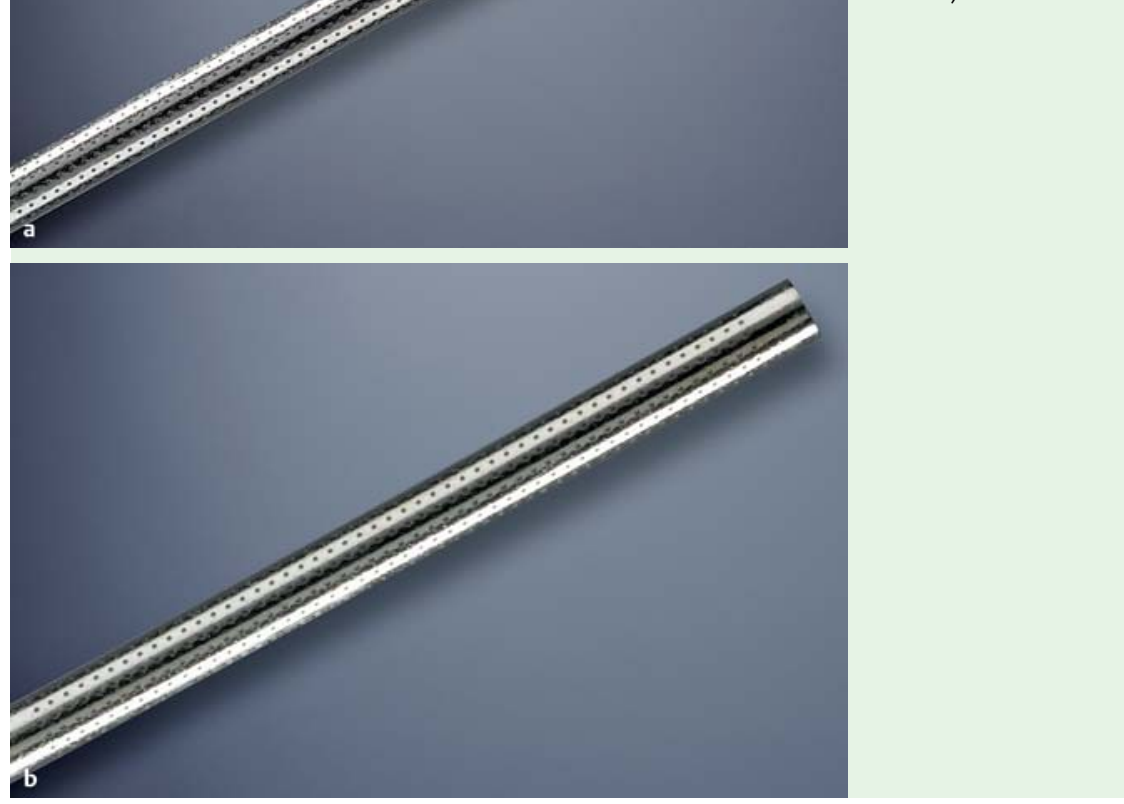

linear echoendoscope (Olympus America, Melville, New York, USA) was used to visualize the upstream bile duct through the duodenal bulb. A puncture was made with the device and the stylet was withdrawn. With a 0.035 -inch guide wire in the intrahepatic duct, a $10 \times 60-\mathrm{mm}$, covered metal stent (Wallflex; Boston Scientific, Natick, Massachusetts, USA) was inserted, after passage of a 7-Fr stepdilator ( Video 1). The patient was discharged home feeling well the next day.

The second patient was a 52-year-old man with jaundice due to tumor progression after chemoradiotherapy for an inoperable tumor of the body of the pancreas. Two attempts at conventional access by different physicians had resulted in failure to get a wire through the stricture; as a result it seemed unlikely that a rendezvous procedure would succeed. The same echoendoscope was used to access the upstream duct through the bulb. The needle stylet was withdrawn; a 7-Fr stepdilator was then passed over a wire, followed by a covered $10 \times 60-\mathrm{mm}$ metal stent (Viabil;
Gore, Flagstaff, Arizona, USA) to drain the bile duct into the duodenum. Of note, the inner catheter would not come back through the waist of the stent, which required balloon dilation. The patient felt well the next morning and was discharged.

In both cases the wire exchanges were smooth, without trauma or stripping of the wire coating, arguably representing a major improvement over the conventional needles in this regard. The needle puncture did not appear any more difficult than with a traditional $19-G$ needle; echogenicity of the sheath appeared excellent ( Video 1). Care must be taken to ensure the catheter tip, and not just the tip of the

\section{Video 1}

This video demonstrates an endoscopic ultrasound (EUS)-guided puncture of a dilated bile duct upstream from an obstructing duodenal cancer, followed by dilation of the tract, and placement of a covered metal stent across the choledochoduodenostomy. 
needle stylet, is in the duct before the stylet is withdrawn. In addition, if the catheter were to fall back out of the duct, it would not be possible to repuncture the bile duct without reinserting the sharp stylet as the device tip is blunt. Although foreign bodies are known to occur with conventional needles $[3,4]$, their exact rate of formation is unknown, as reporting has not been systematic. Rates may however be higher following rendezvous procedures, which typically require more manipulation of the wire for the stricture/papilla to be traversed.

\section{Endoscopy_UCTN_Code_TTT_1AS_2AG}

Competing interests: The author has received honoraria from Olympus America for acting as a consultant on endoscopy reporting software and as a moderator of an endoscopy; he also has received honoraria for speaking at continuing medical education events from Cook Medical.

\section{J. Romagnuolo, MD, MSc, Professor of Medicine}

Medical University of South Carolina,

Charleston, South Carolina, USA

\section{References}

1 Kemper FJ, Tielbeek AV, el Biltagiu $S$ et al. Shearing of the plastic coating of a hydrophilic guide wire in a right femoropopliteal bypass graft: removal from the contralateral side. Cathet Cardiovasc Diagn 1992; 27: 209-211

2 Reddy SC, Kamath P, Talwar KK et al. Guide wire outer coat shearing and embolisation: an unusual complication of pericardiocentesis. Int J Cardiol 1997; 60: 15-18

3 De Witt J, Sherman S, Lillemoe KD. Fracture of an EUS-guided FNA needle during an attempted rendezvous for an inaccessible pancreatic duct. Gastrointest Endosc 2011; 73: $171-173$

4 Perez-Miranda M, de la Serna C, Diez-Redondo P, Vila JJ. Endosonography-guided cholangiopancreatography as a salvage drainage procedure for obstructed biliary and pancreatic ducts. World J Gastrointest Endosc 2010; 2: 212-222
Bibliography

DOI $10.1055 / \mathrm{s}-0030-1256859$

Endoscopy 2011; 43: E356-E357

(c) Georg Thieme Verlag KG Stuttgart · New York . ISSN 0013-726X

Corresponding author

\section{J. Romagnuolo, MD}

MUSC GI and Hepatology

25 Courtenay Drive, ART 7100, MSC 290

Charleston

South Carolina, 29425

USA

Fax: +1-843-876-4261

romagnuo@musc.edu 